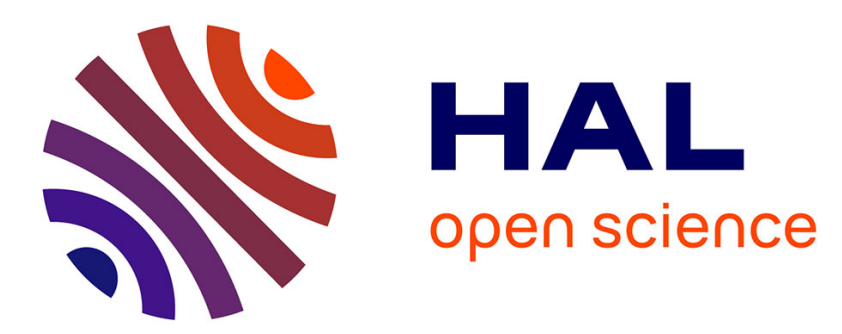

\title{
Which isoplanatic patch size for high angular resolution techniques?
}

\author{
Aziz Ziad, Eric Aristidi, Julien Chabé, Yan Fanteï-Caujolle, Catherine \\ Renaud, Christophe Giordano
}

\section{To cite this version:}

Aziz Ziad, Eric Aristidi, Julien Chabé, Yan Fanteï-Caujolle, Catherine Renaud, et al.. Which isoplanatic patch size for high angular resolution techniques?. SPIE Astronomical Telescopes + Instrumentation, Dec 2020, Online Only, United States. pp.114487O, 10.1117/12.2562252 hal-03122670

\section{HAL Id: hal-03122670 \\ https://hal.science/hal-03122670}

Submitted on 9 Dec 2021

HAL is a multi-disciplinary open access archive for the deposit and dissemination of scientific research documents, whether they are published or not. The documents may come from teaching and research institutions in France or abroad, or from public or private research centers.
L'archive ouverte pluridisciplinaire HAL, est destinée au dépôt et à la diffusion de documents scientifiques de niveau recherche, publiés ou non, émanant des établissements d'enseignement et de recherche français ou étrangers, des laboratoires publics ou privés. 


\title{
Which isoplanatic patch size for High Angular Resolution Techniques?
}

\author{
Aziz Ziad ${ }^{\mathrm{a}}$, Eric Aristidia ${ }^{\mathrm{a}}$,Julien Chabéb, Yan Fantë̈-Caujolle ${ }^{\mathrm{a}}$, Catherine Renaud $^{\mathrm{a}}$, and \\ Christophe Giordano ${ }^{a}$ \\ aUniversité Côte d'Azur, OCA, CNRS, Lagrange, Parc Valrose 06108 Nice Cedex 2, France \\ bUniversité Côte d'Azur, OCA, CNRS, IRD, Géoazur, 2130 route de l'Observatoire, 06460 \\ Caussols, France
}

\begin{abstract}
The isoplanatic patch size is of interest to reach a high performance with an Adaptive Optics system, since the reference star for its calibration should be located in the same isoplanatic domain than the science source. Different techniques and methods have been developed leading to estimations of the isoplanatic patch but all are model-dependent. We first present a review of these techniques and methods of isoplanatic patch size measurement. We developed a new technique for the estimation of the isoplanatic angle based on an extended object. This technique is now part of our new turbulence profile monitor PML based on the observation of the Moon limb or Sun edge. The first statistics of the isoplanatic angle with this new technique are presented and compared to the exiting techniques based on scintillation measurements or other turbulence parameters such as Fried parameter and/or $C_{n}^{2}$ profile.
\end{abstract}

Keywords: Site-testing, atmospheric optics, atmospheric turbulence, turbulence monitoring, adaptive optics, interferometry.

\section{INTRODUCTION}

The atmospheric turbulence parameters have a strong impact on the image formation through the atmosphere. Indeed, The effect of atmospheric turbulence on wavefronts reduces severely the resolution of ground-based astronomical observations. Different High Angular Resolution (HAR) techniques have been developed to achieve diffraction limited resolution of observing instruments, namely Adaptive Optics (AO) and long baseline interferometry (LBI). Design, performance and optimization of these HAR techniques requires a well-knowledge of atmospheric turbulence parameters, notably the seeing (or Fried parameter $r_{0}$ ), the outer scale $\mathcal{L}_{0}$, the coherence time $\tau_{0}$ and the isoplanatic domain $\theta_{0}$. Knowledge of these parameters is also important for future Extremely Large Telescope specifications. Indeed, the Fried parameter and $\mathcal{L}_{0}$ are critical for the deformable mirror in terms of the number of actuators and the required stroke. The knowledge of the wavefront coherence time $\tau_{0}$ is of interest to optimize the HAR technique detector exposure time and the correction frequency of the AO system. In addition, the choice of the AO reference star must take into account the constraints related to the isoplanatic angle. Indeed, a compromise must be found between the magnitude of the reference inside the isoplanatic domain. Different techniques have been used for direct and indirect estimation of the isoplanatic based on different measured quantities such as wavefront phase, Angle-of-Arrival and scintillation. ${ }^{1-4}$ But it is difficult to have analytical relations between the isoplanatic angle definitions corresponding to these different observables.

In this paper we provide the first comparisons of the isoplanatic domaine measured with different instruments using different techniques based on different observables. The first statistics of the non model-dependent isoplanatic angle measured with the PML (Profiler of Moon Limb) instrument ${ }^{5}$ are presented in Sect. 4. These statistics are compared to those provided from the Generalized Seeing Monitor (GSM) database ${ }^{4}$ and the Generalized DIMM (GDIMM) as well. The theoretical background on the isoplanatic angle and particularly a review of the different definitions using different observable quantities is detailed in Sect. 2. Temporal stability of the isoplanatic range is evaluated, compared to other atmospheric turbulence parameters and discussed in Sect. 5.

Further author information: (Send correspondence to A. Z.): E-mail: ziad@unice.fr, Telephone: (33) (0)4 89150392 


\section{THEORETICAL BACKGROUND}

Different techniques are used in the literature for the estimation of the isoplanatic angle using different measured quantities such as wavefront phase, AA fluctuations or scintillation. It is obvious that isoplanatic angles deduced from phase or AA fluctuations are not necessary similar since these quantities are related by a spatial first derivative and therefore the measurement direction (angle of the measured AA with the x-axis) in the case of AA is crucial. In this section, we will summarize the different used definitions of the isoplanatic angle to point out the relevant ones for interferometry and AO systems.

The most known definition of the isoplanatic angle $\theta_{0}$ is the Roddier's one, which is deduced from the spatioangular correlation of phase complex amplitudes of two point sources separated by an angle $\theta .^{1}$ Indeed, the ispolanatic angle is obtained when the phase structure function is equal to $1 \mathrm{rad}^{2}$, leading to,

$$
\theta_{0}=0.31 \frac{r_{0}}{\bar{h}}
$$

where $r_{0}$ represents the Fried parameter and $\bar{h}$ an average (or equivalent) turbulence altitude depending on the vertical distribution of the turbulence characterized by the $C_{n}^{2}$ profile. ${ }^{6}$ This definition is used in the techniques of the most known profilers such as Scidar or radio-sounding balloons to provide estimation of the isoplanatic angle.

On the other hand, the easiest way to measure the isoplanetic angle $\theta_{0}$ is from the scintillation of a single star observed through a pupil of $10 \mathrm{~cm}$ diameter and a central obstruction of $4 \mathrm{~cm}$. The principle of the calculation is based on the similarity of the theoretical expressions of $\theta_{0}$ and the scintillation index. ${ }^{3,4} \theta_{0}$ is obtained in arcsec for a wavelength $\lambda=500 \mathrm{~nm}$ from the following formula,

$$
\theta_{0}^{-5 / 3}=A(\cos z)^{-8 / 3} s
$$

where $A=14.87$ is computed numerically from eqs. 19 and 21 of Ref., ${ }^{4} z$ is the zenithal distance of the star.

In addition, another possibility of a direct measurement of the isoplanatic angle exists, consisting of the use of an extended object. Indeed, the structure function of AA fluctuations along the Moon limb or the Sun edge is very efficient to provide estimation of the isoplanatism range. ${ }^{6}$ The wavefront isoplanatic angle $\theta_{0, \alpha}$ is derived from the structure function of AA fluctuations $D_{\alpha}(\theta)$, in a similar way than in Ref. ${ }^{7}$ for the AA coherence time. Hence, we define $\theta_{0, \alpha}$ as the angle for which $D_{\alpha}(\theta)$ is equal to the $\frac{1}{\kappa}$ of its saturation value, with $\kappa$ a constant.

Examples of angular structure functions of AA fluctuations $D_{\alpha}(\theta)$ deduced from Sun edge are shown in Fig. 1 of Ref., ${ }^{6}$ obtained with the PML instrument at the Calern Observatory. The saturation level of $D_{\alpha}(\theta)$ is given by AA variance which is deduced from Eq. 8 of Ref. ${ }^{6}$ for $\theta=0$. The fold of the saturation by a constant $\kappa=e$ lead to $\theta_{0, \alpha}=7.7^{\prime \prime}$. Including this value of $\theta_{0, \alpha}$ in Eqs 11 and 12 of Ref. ${ }^{6}$ and assuming an outer scale of $20 \mathrm{~m}$, one can deduce the equivalent altitude $(\bar{h}=2897 \mathrm{~m})$. Combining this value of $\bar{h}$ with the seeing measured also from PML $\left(2.59^{\prime \prime}\right.$ at $\left.\lambda=0.5 \mu \mathrm{m}\right)$ in Eq. 1, one obtain an estimation of isoplanatic angle $\left(\theta_{0}=0.92^{\prime \prime}\right)$. While the

value obtained from the $C_{N}^{2}$ profile measured simultaneously by the PML instrument ${ }^{6}$ is of $\theta_{0}=1.03^{\prime \prime}$. These values are in good agreement proving the coherence of these techniques.

\section{INSTRUMENTS OF ISOPLANATIC DOMAIN ESTIMATION}

\subsection{PML instrument}

The PML (Profiler of Moon Limb) instrument is dedicated to the extraction of the $C_{n}^{2}$ profile with high vertical resolution from lunar (or solar) limb fluctuations. The PML instrument is based on a differential method by observation of the lunar (or solar) limb through two sub-apertures (Fig. 1). The Moon or Sun limb acts as a continuum of double stars with all possible angular separations required between two points to scan the atmosphere with a very fine resolution.

The PML instrument consists of a 16-inch telescope (Meade M16) installed on an Astrophysics AP3600 mount. The pupil mask, composed of two sub-apertures of diameter $D=6 \mathrm{~cm}$ separated by a baseline $B=26.7$ $\mathrm{cm}$, is placed at the entrance pupil of the telescope (Fig. 1). An optical system is installed at the ouput of the 


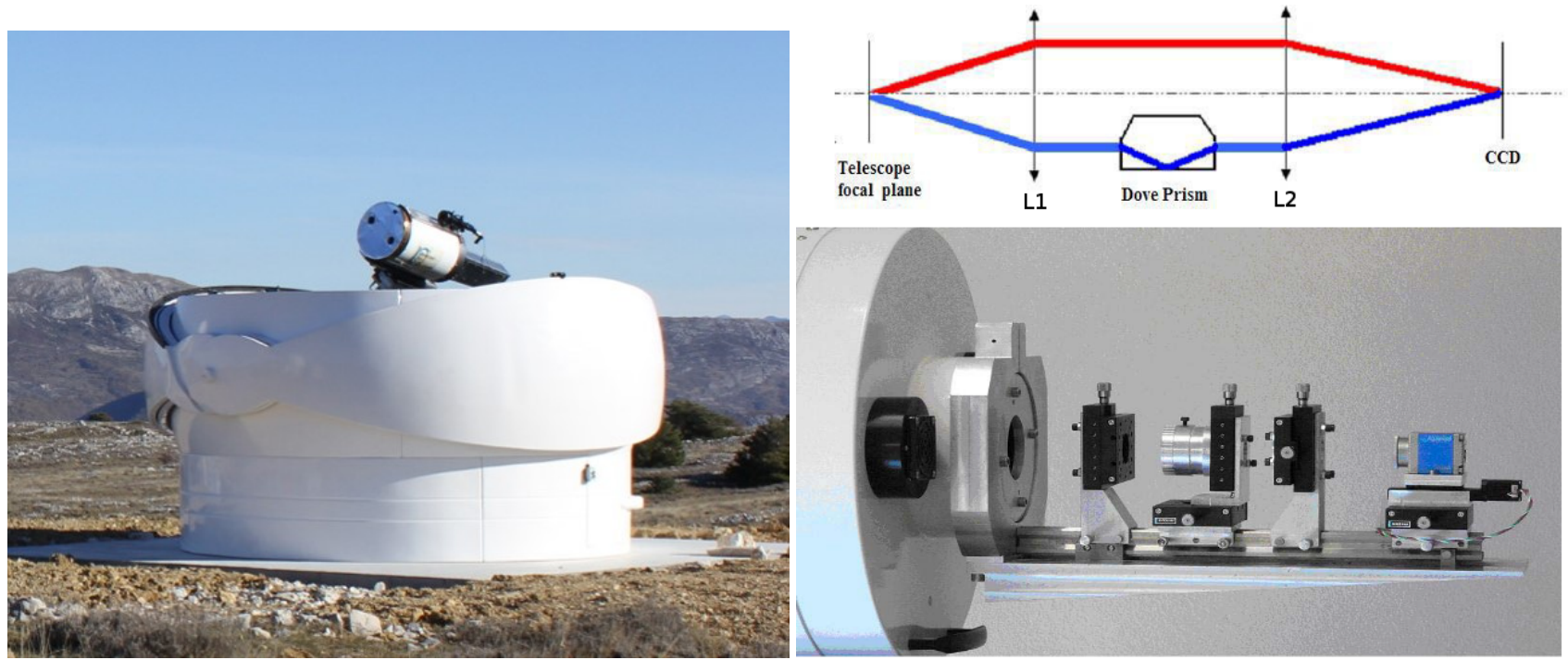

Figure 1. Left: the Profiler of Moon Limb instrument inside its 12ft AllSky dome. Right: The optical device of the PML instrument.

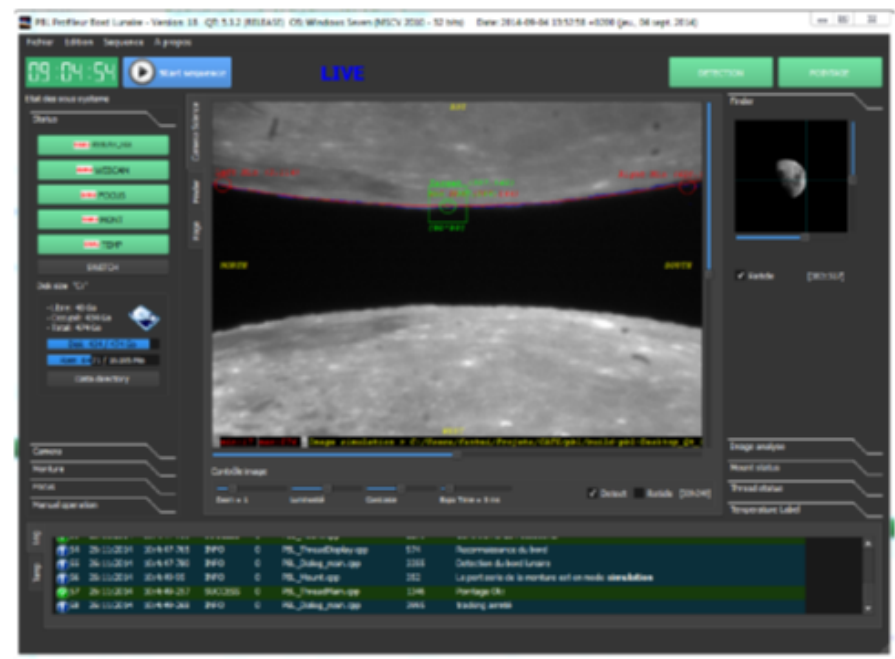

Figure 2. The graphical user interface of the PML instrument showing the two Moon limb images.

telescope. It consists of a collimated beam by using a first lens L1 placed at its focal length from the telescope focus (Fig. 1). Then, two parallel beams are formed at the output of L1 corresponding to each sub-aperture. A Dove prism is inserted on one of the two beams to reverse one of two images of the lunar (solar) edge in order to avoid an overlapping of the two images of the Moon/Sun (Fig. 2). A second lens L2 is used to form the two images of the Moon limb (or Sun edge) on the CCD camera. Each optical element is placed on a micro-control plate allowing fine adjustments. To compensate for variations in the telescope's focus because of the temperature variations, we installed the CCD camera on an automatic micro-control plate controlled by the acquisition software (Fig. 1). Images at the focal plane are recorded using a PixelFly CCD camera with $640 \times 480$ pixel matrix. In order to freeze atmospheric effects on the motion of the Moon's (or Sun) limb image and to have enough flux, the exposure time is set to $5 \mathrm{~ms}$.

The principle of the PML instrument is based on the measurement of the angular correlation of the fluctuation differences in the wavefront AA deduced from the motion of the Moon's (or Sun) limb image. The AA fluctuations are measured perpendicularly to the lunar (or solar) limb leading to transverse correlations for different angular separations along the Moon (or Sun). The observed Moon or Sun limb is parallel to the sub-apertures baseline. 
In this case, the transverse covariance of the difference of the AA fluctuations (motion of the Moon or Sun limb) $\alpha$ between the two images of the lunar (or solar) limb (Fig. 2) corresponds to

$$
C_{\Delta \alpha}(\theta)=\left\langle\left[\alpha\left(r, \theta_{0}\right)-\alpha\left(r+B, \theta_{0}\right)\right]\left[\alpha\left(r, \theta+\theta_{0}\right)-\alpha\left(r+B, \theta+\theta_{0}\right)\right]\right\rangle
$$

where $\alpha\left(r, \theta_{0}\right)$ and $\alpha\left(r, \theta+\theta_{0}\right)$ represent the fluctuations of the lunar (or solar) limb image observed through the first subaperture of the PML and measured at the angular positions $\theta_{0}$ and $\theta+\theta_{0}$, respectively. While, $\alpha\left(r+B, \theta_{0}\right)$ and $\alpha\left(r+B, \theta+\theta_{0}\right)$ are the measured fluctuations corresponding to the second subaperture. We assume space invariance of fluctuation differences, so that $\theta_{0}$ can be taken to 0 without loss of generality. This angular covariance is given by, ${ }^{5,8}$

$$
C_{\Delta \alpha}(\theta)=\int d h C_{N}^{2}(h) K_{\alpha}(B, h, \theta)
$$

where the kernel $K_{\alpha}(B, h, \theta)=2 C_{\alpha}(\theta h)-C_{\alpha}(B-\theta h)-C_{\alpha}(B+\theta h)$ is a triplet of normalized spatial covariances which in the case of the von Kàrmàn model for a baseline $\varrho$, a sub-aperture diameter $\mathrm{D}$ (here $6 \mathrm{~cm}$ ), and a single layer at altitude $h$.

The spatial covariance triplet above is similar to the Scidar's one. ${ }^{9}$ The position of the lateral peak defines the altitude of the turbulent layer $(B= \pm \theta h)$ so that its height leads to the contribution of this layer to the $C_{N}^{2}(h)$ profile. For the whole atmosphere we have a superposition of different triplets corresponding to different turbulent layers (see Fig. 6 of $^{5}$ as an example of illustration).

The spatial covariance $C_{\alpha}$ is non-linear but analytical solution of the integral expression are given by ${ }^{10}$ through the use of Mellin transform. Resulting covariances are simplified into series or finite solutions. Approximations however are different depending on the baseline length. We used these approximations and the assumption of a discrete turbulent profile to transform eq. 4 into

$$
C_{\Delta \alpha}(\theta)=\sum \Delta h_{i} C_{N}^{2}\left(h_{i}\right) \widehat{K}_{\alpha}\left(B, h_{i}, \theta\right)
$$

where $\widehat{K}_{\alpha}\left(B, h_{i}, \theta\right)$ is the modified spatial covariance triplet and $\Delta h_{i}$ is the thickness of the layer i. This equation takes a matrix form: $Y=M . X$ where $\mathrm{X}$ and $\mathrm{Y}$ are both vectors corresponding respectively to the sampled $C_{N}^{2}\left(h_{i}\right)$ and the covariance difference $C_{\Delta \alpha}(\theta)$, the matrix $\mathrm{M}$ contains the modified spatial covariance triplet weighted with $\Delta h_{i}: M=\widehat{K}_{\alpha}\left(B, h_{i}, \theta\right) . \Delta h_{i}$.

The $C_{N}^{2}(h)$ profiles are retrieved by solving an inverse problem via minimization of a maximum likelihood criterion under positivity constraint using an iterative gradient method. ${ }^{11,12}$ The minimization process makes use of a regularization constraint based on the gradient. A full description of the algorithm is given in Ref. ${ }^{8}$ In addition, we also used a simulated annealing algorithm for the minimization process leading to the reconstruction of the turbulence profile $C_{N}^{2}(h)^{13,14}$ but it appeared to be very slow and not suitable for real-time profile calculation.

The PML acquisition software provides a real time computation of the $C_{n}^{2}$ profile with a high resolution vertical every 2 minutes. In addition, the PML instrument is now equipped with automatic panels to cover the two subapertures with solar filters for a fast and automatic switch from night/Moon observation to day/Sun observation. ${ }^{12}$ We then have a unique instrument to study the turbulence conditions at the daytime to nighttime transition as you can see in Fig. 4. Other parameters of turbulence are also accessible from this instrument such as the profile of outer scale, the seeing, and the isoplanatic angle. For this latter, two techniques described in section 2 are used. The first and easiest one, is based on the $C_{n}^{2}$ profile to deduce first the $\bar{h}$ from Eq. 3 of Ref. ${ }^{6}$ which is introduced into Eq. 1 to provide an estimation of $\theta_{0}$. The second method is based on the angular structure functions of AA fluctuations $D_{\alpha}(\theta)$ deduced from Moon (or Sun) limb as shown in Fig. 1 of Ref. ${ }^{6}$ The saturation level is obtained directly from $D_{\alpha}(\theta)$ for large angular separations $\theta$ as shown in Fig. 1 of Ref. ${ }^{6}$ The fold of $D_{\alpha}(\theta)$ by a constant $\kappa=e$ lead to $\theta_{0, \alpha}$. Including this value of $\theta_{0, \alpha}$ in Eqs. 11 or 12 of Ref., ${ }^{6}$ the equivalent altitude $\bar{h}$ is obtained. Then, Eq. 1 , gives the isoplanatic angle $\theta_{0}$ estimation. 

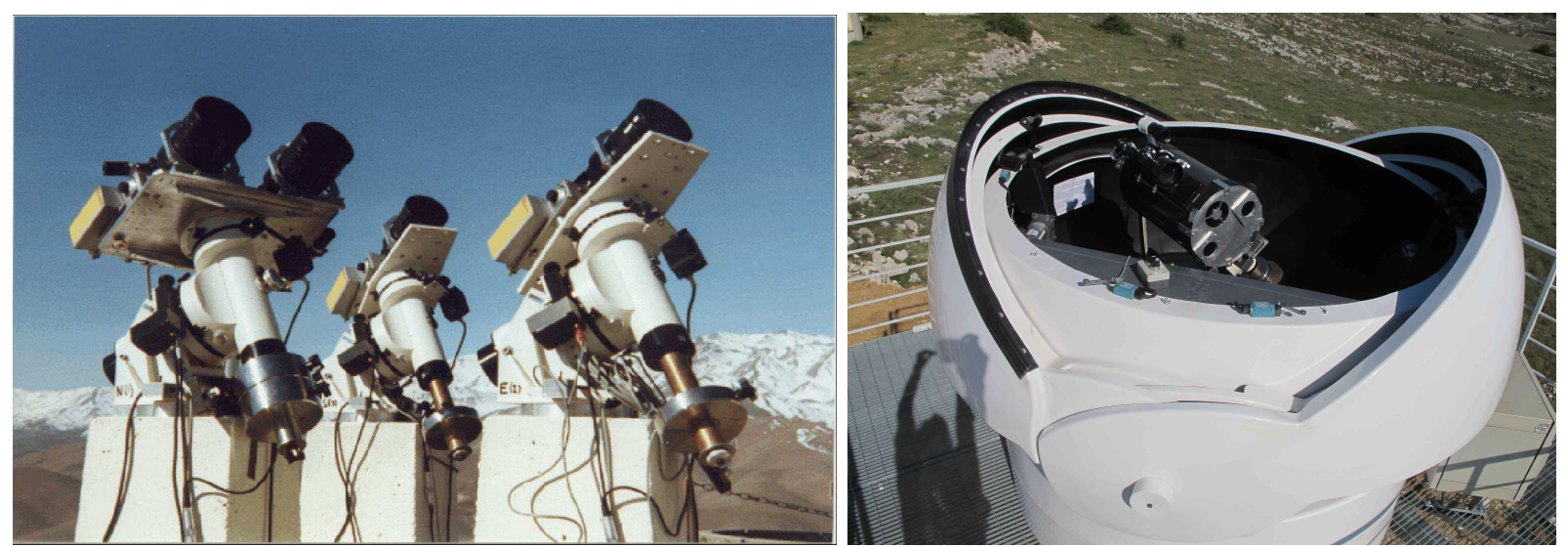

Figure 3. Left: standard GSM working layout at La Silla (Chile) Sept. 1997. The GDIMM instrument inside its $7 \mathrm{ft}$ AllSky dome (right) at the Calern Observatory.

\subsection{GSM \& GDIMM instruments}

The GSM instrument (Generalized Seeing Monitor) allows the evaluation of turbulence parameters of the perturbed wavefront by measuring AA fluctuations. Indeed, the GSM uses the same principle as a Shack-Hartmann, i.e., measuring AA at different points of the wavefront and computing AA spatio-temporal correlations. These latters lead to estimates of the seeing $\epsilon_{0}$, outer scale $\mathcal{L}_{0}$ and coherence time $\tau_{0}$ when scintillation with a special spatial filtering provide isoplanatic angle $\theta_{0}$ estimations.

The instrument consists of four 10-cm telescopes on equatorial mounts equipped (Fig 3) with detection modules measuring the AA fluctuations and interfaced to a computer PC managing simultaneously the 4 modules. Each telescope, pointing at the same star, measures the AA fluctuations by means of flux modulation which is produced by the displacement of the star image over a Ronchi grating. ${ }^{4}$ Two telescopes are installed on a common mount on a central pillar working as a differential image motion monitor $\left(\mathrm{DIMM}^{15}\right)$ with a $25 \mathrm{~cm}$ baseline (distance between two GSM modules). Two other telescopes have different mounts on separate pillars, located $0.8 \mathrm{~m}$ to the south and $1 \mathrm{~m}$ to the east from the central pillar, thus forming an L-shaped configuration which has been chosen for more sensitivity to the outer scale. The telescopes were generally situated $1.7 \mathrm{~m}$ above the ground.

The AA fluctuations are measured with $5 \mathrm{~ms}$ resolution time during continuous sequences of 2 minutes. Data are processed immediately after each acquisition, allowing quasi real-time monitoring of the turbulence parameters. Sequences are repeated typically every 4 minutes.

The AA covariances are computed for each baseline ( 6 baselines with 4 GSM modules) and normalized by the differential variance of $\mathrm{AA}$ on the $25-\mathrm{cm}$ baseline. They are compared to von Kàrmàn theoretical normalized covariances ${ }^{16}$ and the appropriate $\mathcal{L}_{0}$ is found for each baseline. The final value of $\mathcal{L}_{0}$ is taken as the median of the 6 individual $\mathcal{L}_{0}$ values and its error is estimated. The seeing $\epsilon_{0}$ is calculated from the differential variance given by the coupled modules as in the DIMM instrument. ${ }^{15}$ The scintillation index $s$ is computed during data reduction and an estimate of the isoplanatic angle is deduced from Eq. 2. ${ }^{4}$

A quantification of the different sources of GSM noise has been performed and hence corrections of photon and scintillation noises are done before data processing. Another correction for finite exposure time is also performed; it consists in computing AA or scintillation statistical moments for $5 \mathrm{~ms}$ and $10 \mathrm{~ms}$ and in extrapolating linearly to the $0 \mathrm{~ms}$ exposure time. Indeed, GSM measures AA and intensity with $5 \mathrm{~ms}$ resolution and two successive measurements are adjacent. Then by bining two successive AA or intensity measurements, we get 10ms exposure time AA and intensity estimations. Finally, the statistical errors of the computed variances and covariances are estimated and consequently the errors of the turbulence parameters measured with GSM, including the isoplanatic angle are provided. 
Since 1997 and for more than 15 years, GSM was used in a large number of astronomical observatories and for prospecting potential new sites. ${ }^{4}$ Table 1 summarizes statistics of the seeing and isoplanatic angle measured by GSM.

Recently, we developed a new monitor, the Generalized Differential Image Motion Monitor, a compact instrument aiming at replacing the aging GSM. ${ }^{12,17,18}$ GDIMM is very similar to a DIMM, with 3 sub-apertures instead of 2. The seeing is obtained by the DIMM method, using two sub-pupils of same diameter. The third aperture has a diameter of $10 \mathrm{~cm}$ with $4 \mathrm{~cm}$ central obstruction, allowing to estimate the isoplanatic angle via the scintillation as in the GSM instrument. The GDIMM instrument uses a CCD detector with an exposure time of $5 \mathrm{~ms}$ and correction of exposure time effect is obtained by extrapolation using 5 and $10 \mathrm{~ms}$ exposures. GDIMM is a part of the Calern Atmospheric Turbulence Station (CATS), ${ }^{12,19}$ who was developed as a site monitoring facility at the Plateau de Calern (France). The instrument is running routinely since the end of 2015 and provides a measurement of turbulence parameters every 2 minutes. Statistics of the isoplanatic angle as measured by GDIMM at Calern observatory are presented in Table 1.

Between 2004 and 2012, a set of instruments were developed to monitor turbulence parameters on the site of Dome C, Antarctica (see ${ }^{20}$ and reference therein). A new version of the GSM, dedicated to extremely cold conditions was part of these instruments. ${ }^{21}$ As for GDIMM and initial GSM , this antarctic GSM version allowed to estimate the isoplanatic angle by means of scintillation. Several years of monitoring resulted in a large amount of data in various conditions (winter and summer). Statistics for the seeing and the isoplanatic angle obtained with this antarctic GSM are presented in Table 1
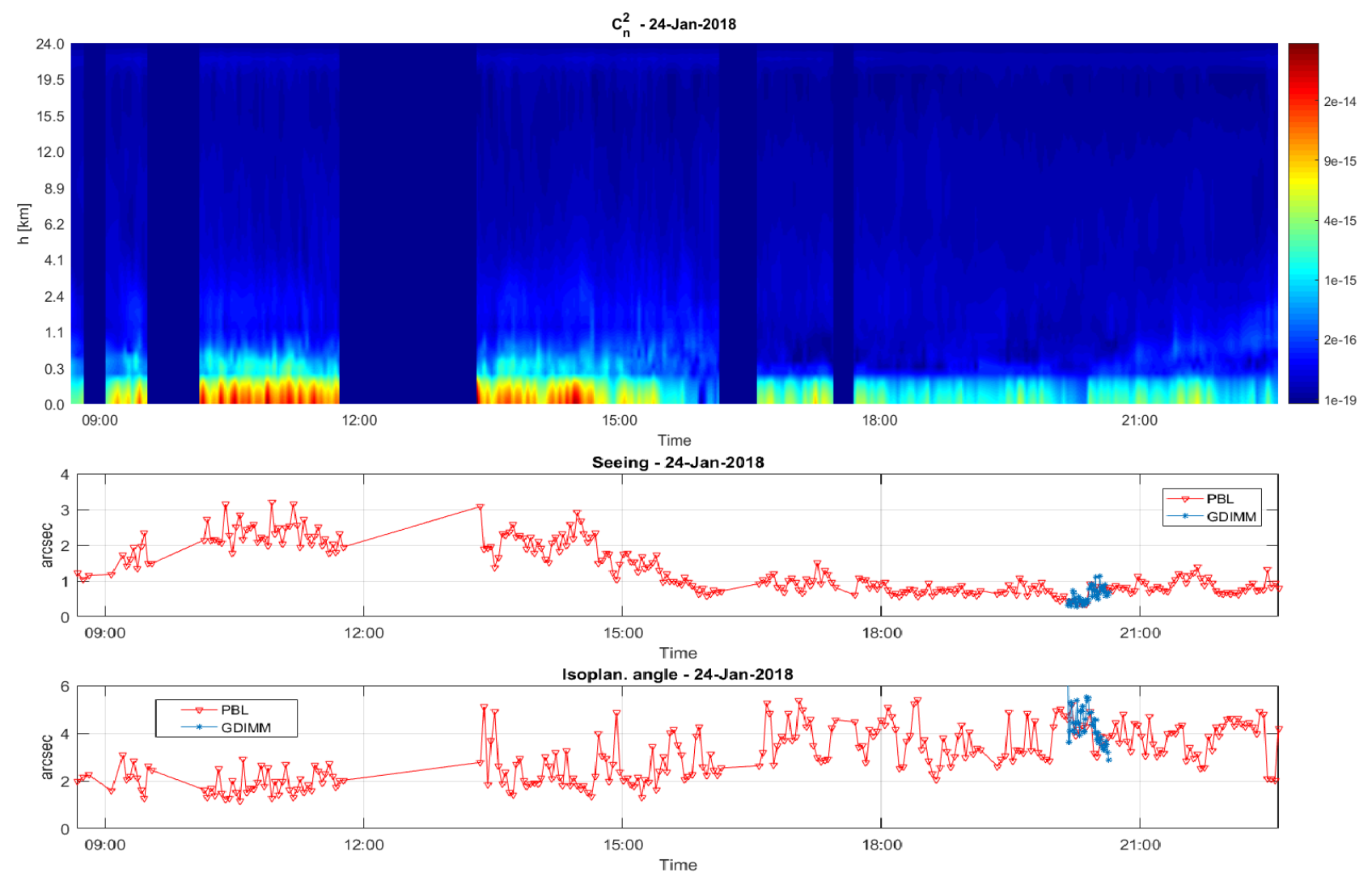

Figure 4. Turbulence profiles of January 24th, 2018 at the Calern Observatory obtained with the PML instrument (Top). The altitude is given above the Calern Observatory $(1270 \mathrm{~m})$. Observations have obtained on the Sun edge before 5PM and on the Moon limb just after. Bottom: Comparison of seeing and isoplanatic results obtained from the PML and GDIMM measurements for the same night. The isoplanatic PML results are obtained using the $C_{N}^{2}$ profiles when GDIMM $\theta_{0}$ is deduced from scintillation. 


\section{ISOPLANATIC DATA \& COMPARISONS}

In this section, we are going to show results of the different techniques presented above, leading to the estimation of the isoplanatic angle. Indeed, estimation of $\theta_{0}$ from the GSM and GDIMM instruments using scintillation measurements are presented. In addition, estimations of the isoplanatic angle from $C_{N}^{2}$ profile using different instrument such as PML and radio-sounding balloons are also presented here-after. Results of a new technique based on angular structure function (sect. 2) leading to a non-model dependent estimation of $\theta_{0}$, are also presented. Comparisons of simultaneous measurements of $\theta_{0}$ from these different techniques on different sites over the world are shown.
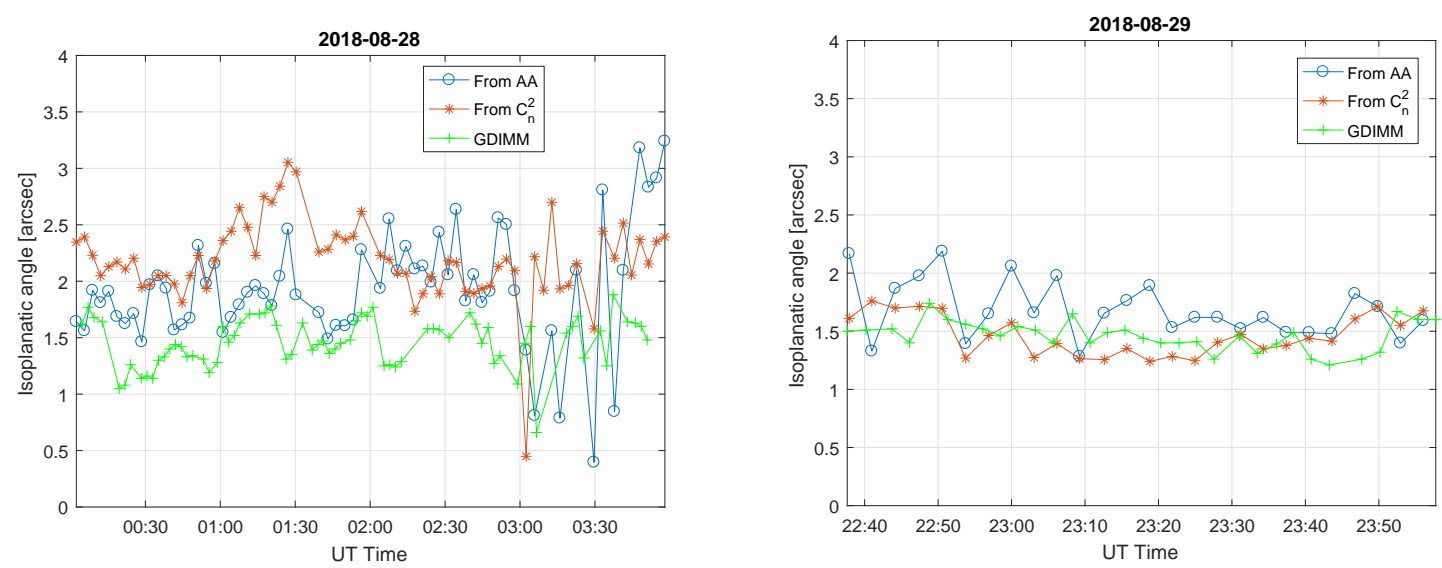

Figure 5. Comparison of isoplanatic results obtained from the PML and GDIMM measurements at the Calern Observatory. The PML results are obtained using the $C_{N}^{2}$ profiles (red) and AA structure function (blue) when GDIMM $\theta_{0}$ is deduced from scintillation (green).

Fig. 4 shows an example of the turbulence profile evolution with the PML throughout the daytime and the nighttime of January 24th, 2018 at the Calern Observatory. The figure shows the full PML profiles with 33 layers from the Sun until 5PM and from the Moon just after. The resolution obtained by the PML is $\Delta h=100 \mathrm{~m}$ for the ground layer $(h \leq 1 \mathrm{~km}), \Delta h=500 \mathrm{~m}$ for the low free atmosphere $(1 \mathrm{~km}<h<5 \mathrm{~km}), \Delta h=1000 \mathrm{~m}$ for the mid-free atmosphere $(5 \mathrm{~km}<h<15 \mathrm{~km})$, and $\Delta h=2000 \mathrm{~m}$ for the high free atmosphere $(h>15 \mathrm{~km})$. The highest altitude $h_{\max }$ reachable with the PML is more than $50 \mathrm{~km}$ but we limited $h_{\max }$ to $25 \mathrm{~km}$ since the turbulence is very low above this altitude. On the other hand, because of a limited field of view the PML instrument has a minimum altitude detectable which is around $100 \mathrm{~m}$. The contribution of the lowest layer $0-100 \mathrm{~m}$ is obtained by the difference between the profile deduced from the inversion of the PML covariances and the total seeing from DIMM method (sect. 3) using PML data. For the total seeing obtained from PML, we have about 620 estimations (each point of the Moon limb leads to a DIMM measurement) and we keep only the median one (Middle panel of 4). These PML profiles are used to provide the isoplanatic angle estimations as shown in the bottom panel of Fig. 4. As one can see, the nighttime isoplanatic values are better than the daytime because the seeing conditions are progressively better when passing from Sun to Moon observations. But usually it happens that daytime and nighttime $\theta_{0}$ are comparable since $\theta_{0}$ is dominated by the high layers in the atmosphere which are similar for daytime and nighttime conditions because they are depending on large scale meteorological phenomena. On the other hand, on can also remark that isoplanatic results deduced from scintillation (GDIMM data) are comparable to those obtained from PML $C_{N}^{2}$ profile. This result is not a general tendency as shown in Fig. 5. Indeed, one can notice that $\theta_{0}$ estimations from PML using $C_{N}^{2}$ profiles, AA structure function and scintillation could be very close for some nights as shown for August 29th. Scintillation data for some nights underestimate $\theta_{0}$ as shown for August 28th. For this night, the scintillation and seeing were worse leading to large intensity and AA fluctuations, respectively and making their detection easiest and precise with PML and GDIMM instruments. Other error source of scintillation measurement, is the presence of the Moon in the same direction in the Sky than the observed star. Indeed, when the Moon was illuminating directly the pupil of the GDIMM, this induced fluctuations in the intensity of the spots on the CCD camera. 


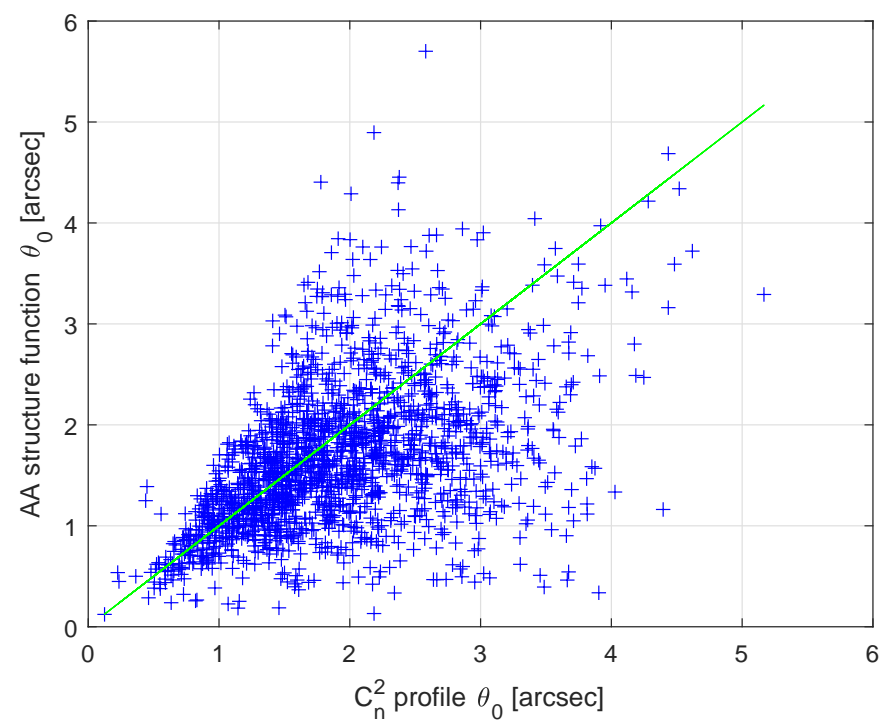

Figure 6. Comparison of isoplanatic results obtained from the PML data using AA structure function method and $C_{N}^{2}$ profiles at the Calern Observatory for the period of September/October 2018. 1830 data are represented, consisting of 300 measurements on Moon limb and 1500 on Sun edge observations. .

These intensity fluctuations are added to those induced by the atmosphere and leading to a reduced $\theta_{0}$. In general, $\theta_{0}$ obtained from the three techniques by means of PML and GDIMM data are coherent. In addition, Fig. 6 shows a comparison of isoplanatic results obtained from the PML data using both techniques based on AA structure function method and $C_{N}^{2}$ profiles. These measurements have been obtained at the Calern Observatory for the period of September/October 2018, consisting of 1830 data distributed between Moon limb and Sun edge observations. On can note that there is a good agreement for small $\theta_{0}$ values and progressively the discrepancy is larger when the isoplanatic angle tends to large values. Good turbulence conditions reduce AA fluctuations and making their detection with the PML less precise, particularly for the AA structure function reconstruction.
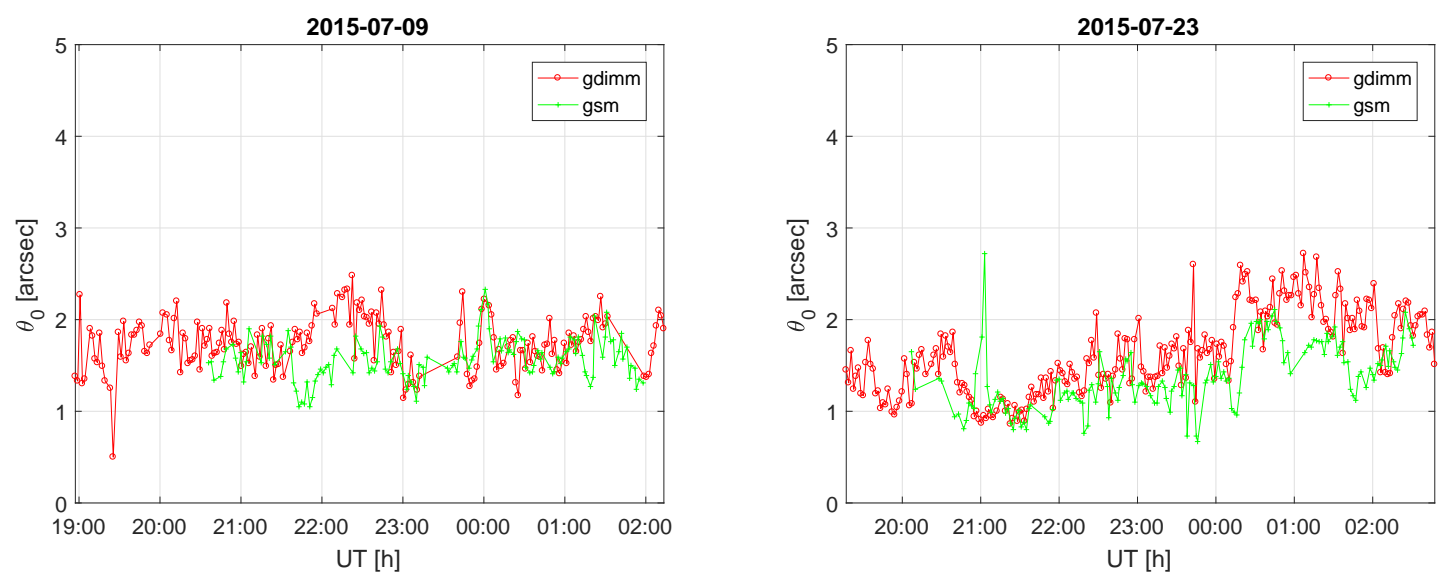

Figure 7. Comparison between GSM and GDIMM isoplanatic data deduced from scintillation measurement at the Calern Observatory in 2015 .

Another example of comparison of different estimation techniques for $\theta_{0}$ was made for the Dome $\mathrm{C}$ site in Antarctica. Profiles of $C_{N}^{2}$ from PML and radio-sounding balloons are presented in Ref. ${ }^{5}$ on Moon limb and Sun edge. From the Moon limb results, one can deduce a Fried parameter $r_{0}=8.2 \mathrm{~cm}$ and the outer scale deduced 
from the GSM instrument was of $\mathcal{L}_{0}=9.1 \mathrm{~m}$. The fold of $D_{\alpha}(\theta)$ by a constant $k=e$ lead to $\theta_{0, \alpha}=9.9^{\prime \prime}$. Including this value of $\theta_{0, \alpha}$ in Eq. 11 of Ref. ${ }^{5}$ using $r_{0}$ and $\mathcal{L}_{0}$ above, we obtained an equivalent altitude $\bar{h}=1024 m$, while values obtained from balloon and PML profiles in Fig. 1 of Ref. ${ }^{5}$ and using Eq. 3 of Ref. $^{6}$ is of $\bar{h}_{\text {Bal }}=805 \mathrm{~m}$ and $\bar{h}_{P M L}=847 \mathrm{~m}$, respectively. Even if the balloon value is smaller for the simple reason that they are not obtained in the same period than the PML results, these estimations are coherent. Using Eq. 1, these equivalent altitudes lead to isoplanatic angles equal respectively to $\theta_{0}=5.13^{\prime \prime}, \theta_{0, B a l}=5.32^{\prime \prime}$ and $\theta_{0, P M L}=6.20^{\prime \prime}$ at $\lambda=0.5 \mu \mathrm{m}$. The median value of the isoplanatic angle at Dome $\mathrm{C}$ statistics deduced from GSM instrument is of $(3.9 \pm 2.4)^{\prime \prime}$ (Table 1) for the whole year but it is lager in the summer season corresponding to the period of PML results above. ${ }^{20}$ Then, all these measurements of the isoplanatic angle at Dome $\mathrm{C}$ from different techniques and instruments are coherent.

Table 1. Data summary of seeing and isoplanatic angle measured by GSM and GDIMM instruments on different sites over the world. Each campaign is characterized by the mean values and standard deviations of seeing and $\theta_{0}$ at $\lambda=0.5 \mu m$ and at the zenith. All instruments extract $\theta_{0}$ from scintillation

\begin{tabular}{|c|c|c|c|c|}
\hline Site & Date & $\begin{array}{l}\text { Number } \\
\text { of data }\end{array}$ & $\begin{array}{l}\text { Seeing } \\
\varepsilon_{0}(\text { as })\end{array}$ & $\begin{array}{l}\text { Isoplanatic } \\
\theta_{0}(a s)\end{array}$ \\
\hline $\begin{array}{l}\text { La Silla } \\
\text { (Chile) }\end{array}$ & $\begin{array}{l}08 / 26 / 97 \\
09 / 20 / 98\end{array}$ & 1422 & $1.64 \pm 0.47$ & $1.25 \pm 0.42$ \\
\hline $\begin{array}{l}\text { Oukaïmeden } \\
\text { (Morocco) }\end{array}$ & $\begin{array}{l}04 / 10 / 98 \\
04 / 25 / 98\end{array}$ & 643 & $1.37 \pm 0.53$ & $1.58 \pm 0.82$ \\
\hline $\begin{array}{l}\text { Maidanak } \\
\text { (Uzbekistan) }\end{array}$ & $\begin{array}{l}07 / 16 / 98 \\
07 / 25 / 98\end{array}$ & 846 & $0.69 \pm 0.25$ & $2.47 \pm 0.71$ \\
\hline $\begin{array}{l}\text { Cerro Pachon } \\
\text { (Chile) }\end{array}$ & $\begin{array}{l}10 / 02 / 98 \\
10 / 09 / 98\end{array}$ & 616 & $0.89 \pm 0.46$ & $2.71 \pm 0.97$ \\
\hline $\begin{array}{l}\text { Cerro Paranal } \\
\text { (Chile) }\end{array}$ & $\begin{array}{l}11 / 27 / 98 \\
12 / 20 / 98 \\
\end{array}$ & 1884 & $0.91 \pm 0.41$ & $1.91 \pm 0.68$ \\
\hline $\begin{array}{l}\text { San Pedo } \\
(\text { Mexico })\end{array}$ & $\begin{array}{l}12 / 03 / 00 \\
12 / 13 / 00\end{array}$ & 572 & $1.15 \pm 0.69$ & $2.04 \pm 0.89$ \\
\hline $\begin{array}{l}\text { Mont Palomar } \\
(U S A)\end{array}$ & $\begin{array}{l}09 / 04 / 00 \\
09 / 13 / 00\end{array}$ & 1089 & $1.49 \pm 0.40$ & $1.96 \pm 0.74$ \\
\hline $\begin{array}{l}\text { Mauna Kea } \\
(\text { Hawaii, USA) }\end{array}$ & $\begin{array}{l}11 / 15 / 01 \\
12 / 04 / 01 \\
\end{array}$ & 726 & $0.75 \pm 0.64$ & $2.94 \pm 0.96$ \\
\hline $\begin{array}{l}\text { Calar Alto } \\
\text { (Spain) }\end{array}$ & $\begin{array}{l}05 / 25 / 02 \\
05 / 30 / 02 \\
\end{array}$ & 487 & $1.41 \pm 0.53$ & $2.27 \pm 0.88$ \\
\hline $\begin{array}{l}\text { La Palma } \\
\text { (Spain) }\end{array}$ & $\begin{array}{l}09 / 04 / 02 \\
09 / 12 / 02\end{array}$ & 676 & $1.14 \pm 0.29$ & $2.13 \pm 0.65$ \\
\hline $\begin{array}{l}\text { Dome C } \\
(\text { Antartica })\end{array}$ & $\begin{array}{l}01 / 01 / 04 \\
05 / 30 / 06\end{array}$ & 46653 & $1.47 \pm 0.80$ & $3.9 \pm 2.40$ \\
\hline $\begin{array}{l}\text { Cerro Paranal } \\
\text { (Chile) }\end{array}$ & $\begin{array}{l}12 / 16 / 07 \\
12 / 25 / 07\end{array}$ & 936 & $1.03 \pm 0.27$ & $2.47 \pm 0.75$ \\
\hline $\begin{array}{l}\text { Calern } \\
\text { (France) }\end{array}$ & $\begin{array}{l}01 / 10 / 17 \\
31 / 10 / 17\end{array}$ & 5260 & $1.44 \pm 0.70$ & $1.96 \pm 0.93$ \\
\hline
\end{tabular}

In 2015, simultaneous data sets of isoplanatic angle were obtained at the Calern observatory from two instruments, GDIMM and GSM. Even if these instruments use the same technique to extract $\theta_{0}$, the scintillation is not measured exactly in the same way from both instruments. The main difference of scintillation measurement is due to the intensity technique and the used detector. Indeed, the GSM uses an interferometric method with a photomultiplier ${ }^{4}$ when the GDIMM measures the intensity of the observed star image in the telescope focal plane with a CCD. This latter has a non homogeneous sensitivity of the pixels which are less sensitive than a photomultiplier; we have to recall that there is a residual drift due to the diurnal movement which implies a drift of images on the CCD matrix pixels. Thus, due to its interferometric technique and its high sensitivity detector, the GSM provides a more precise scintillation measurements and then isoplanatic angle estimation. In addition, the exposure time effect debiasing is more pertinent with the GSM than with the GDIMM. Indeed, 
in the GSM, 10ms exposure intensity are obtained by binning two contiguous $5 \mathrm{~ms}$ exposures. Whilst, the GDIMM starts acquiring a sequence of $\mathrm{N}$ images with $5 \mathrm{~ms}$ exposure and then immediately another sequence with $10 \mathrm{~ms}$ exposure is recorded. Indeed, comparisons between GSM and GDIMM time series in Fig. 7 show that measurements from GSM are more compact, continuous and stable. In general, GDIMM isoplanatism is a bit overestimated, compared to GSM, due to the whole reasons above. Fig. 7 helps to better reflect this difference.

Table 1 shows the whole statistics of isoplanatic angle obtained at different sites with all instrument using scintillation to extract $\theta_{0}$, i.e. GSM and GDIMM. All $\theta_{0}$ histograms of these campaigns are well fitted with log-normal distributions. In terms of median values, one can remark that some sites have better turbulence conditions. But, this conclusion is not representative of the whole behavior of these sites because of the difference in the observation conditions (campaign period and duration). From these GSM and GDIMM data, the Dome C site presents the largest isoplanatic angle but a poor seeing (the seeing is deteriorated by a strong surface layer and the GSM was installed only $3 m$ above the ground). In addition, some sites such Maidanak and Mauna Kea, present good seeing conditions combined to large ispalanatic angles. This is typical of situations when the turbulence in the ground layer is weak leading to small seeing values. These conditions are favorable for the observations with HAR techniques relaxing the constraints on the AO systems and fringe trackers.

\section{TEMPORAL STABILITY OF THE ISOPLANATIC ANGLE}

It is important for HAR techniques to characterize the temporal evolution of optical turbulence parameters. This temporal stability is particularly fundamental to know how long the isoplanatic angle remains coherent. This issue has been analyzed by Ref. ${ }^{22}$ concerning the seeing prediction and for scheduling astronomical programs. To analyze this temporal variability it was suggested to use the normalized difference (ND) of two seeing measurements separated with a time delay $\Delta t .{ }^{22}$ This ND applied to the isoplanatic measurements leads to the dimensionless quantity:

$$
N D(\Delta t)=\left\langle\frac{\left|\theta_{0}(t+\Delta t)-\theta_{0}(t)\right|}{\left[\theta_{0}(t+\Delta t)+\theta_{0}(t)\right]}\right\rangle
$$

where \langle\rangle stands for ensemble average. Fig. 8 shows this $N D$ evolution for measurements at La Silla Observatory in August/September 1997. ${ }^{23}$ The choice of La Silla data is justified by the fact that the temporal stability study of outer scale, seeing and isoplanatic angle requires an important data density and a tight sampling. The GSM data at La Silla have been obtained over 16 nights (1418 data) with an important data density for each night. Indeed, for this first GSM campaign, we obtained during the observations a reduced lost time (star change, clouds, mechanical problems on mounts...). We first calculated the ND function in Eq. 6 for each night and for three parameters (seeing, isoplanatic angle end outer scale). An average over the 16 nights lead to the result presented in Fig. 8. The first remark is that the amplitude of this relative difference in the case of the isoplanatic angle is comparable to the seeing $\epsilon_{0}$ and less important comparatively to the outer scale. This saturated ND-curve in Fig. 8 could be fitted by an appropriate function defined by ${ }^{22}$ as,

$$
N D(\Delta t)=N D_{s}[1-\exp (-\Delta t / \tau)]
$$

where $N D_{s}$ is the saturation value of $N D$ and $\tau$ is the e-folding time. This function was used to fit the data by least squares minimization as is shown in dashed line in Fig. 8. Then, it was found for the $\theta_{0}$ an e-folding time of $\tau_{I}=8.9 \mathrm{~min}$ which is smaller than the seeing one $\tau_{S}=17.2 \mathrm{~min}$ but closer to the outer scale one $\tau_{L}=6.1 \mathrm{~min}$.

\section{CONCLUSIONS}

For the first time comparisons of the isoplanatic angle measurements using different techniques and different observable quantities are now available. These comparisons have been performed using our database of different instruments such as GSM, PML, GDIMM and radio-sounding balloons. Instruments using same scintillation technique such as GSM and GDIMM provide coherent estimations of $\theta_{0}$ but not identical results depending on the detector sensitivity and exposure time correction. A new technique based on angular structure functions of AA fluctuations using the PML instrument on Moon or Sun limbs, is presented. This technique provides 


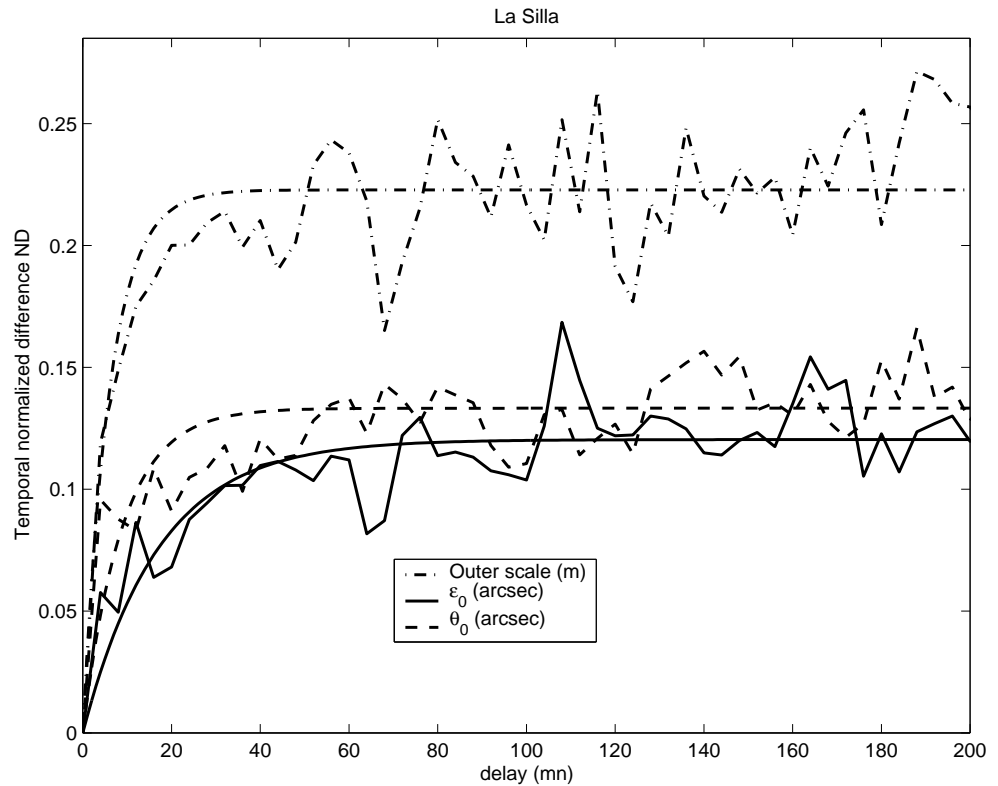

Figure 8. The temporal normalized difference $N D$ for three parameters (seeing, isoplanatic angle and outer scale) obtained with GSM data at La Silla Observatory in August/September 1997. For each parameter, a least-square fit by the model in Eq. 7 is shown.

non model-dependent estimations of isoplanatic angle. In addition, statistics of isoplanatic angle for the major Observatories around the world are presented, thanks the GSM database of atmospheric turbulence parameters. Due to a prevailing surface layer, a large isoplanatic angle at Dome $\mathrm{C}$ in Antarctica is observed making this site to be more favorable for HAR observations. But we have to put this conclusion in perspective due to Dome $\mathrm{C}$ dominant surface layer (SL) reducing the seeing if observations are not performed beyond this SL and also for the extreme conditions of this site. Stability time of the isoplanatic angle appears to be comparable to the outer scale but still two times smaller than the seeing one.

\section{REFERENCES}

[1] Roddier, F., Gilli, J. M., and Lund, G., "On the origin of speckle boiling and its effects in stellar speckle interferometry," Journal of Optics 13(5), 263 (1982).

[2] Fried, D. L. , Opt. Acta 26, 597 (1979).

[3] Loos, G. and Hogge, C., "Turbulence of the upper atmosphere and isoplanatism," Applied Optics 18, 15 (1979).

[4] Ziad, A., Conan, R., Tokovinin, A., Martin, F., and Borgnino, J., "From gsm to gsm," Appl. Opt. 39, 5415 (2000).

[5] Ziad, A., Blary, F., Borgnino, J., Fantë-Caujolle, Y., Aristidi, E., Martin, F., Lanteri, H., Douet, R., Bondoux, E., and Merkarnia, D., "First results of the pml monitor of atmospheric turbulence profile with high vertical resolution," $A \& A$ 559, L6 (2013).

[6] Ziad, A., Aristidi, E., Chabé, J., and Borgnino, J., "On the isoplanatic patch size in high angular resolution techniques," MNRAS 487-3, 3664 (2019).

[7] Ziad, A., Borgnino, J., Ali, W. D., Berdja, A., Maire, J., and Martin, F., "Temporal characterization of atmospheric turbulence with the generalized seeing monitor instrument," Journal of Optics 14(4), 045705 (2012).

[8] Chabé, J., Aristidi, E., Ziad, A., Lantéri, H., Fanteï-Caujolle, Y., Giordano, C., Borgnino, J., Marjani, M., and Renaud, C., "Pml: a generalized monitor of atmospheric turbulence profile with high vertical resolution," Applied Optics 59, 7574 (2020). 
[9] Fuchs, A., Tallon, M., and Vernin, J. PASP 110, 86 (1998).

[10] Conan, R., Modélisation des effets de l'échelle externe de cohérence spatiale du front d'onde pour l'observation à Haute Résolution Angulaire en Astronomie, $\mathrm{PhD}$ thesis, Université de Nice - Sophia Antipolis (2000).

[11] Blary, F., Caractérisation et Modélisation de la Turbulence Optique dans un espace confiné, PhD thesis, Université de Nice Sophia Antipolis (2015).

[12] Ziad, A., Chabé, J., Fanteï-Caujolle, Y., Aristidi, E., Renaud, C., and Ben Rahhal, M., "Cats: an autonomous station for atmospheric turbulence characterization," Astronomical Telescopes + Instrumentation, Proc. SPIE 10703, 107036L (2018).

[13] Maire, J., Ziad, A., Borgnino, J., and Martin, F. MNRAS 377, 232 (2007).

[14] Catala, L., Ziad, A., Fanteï-Caujolle, Y., Crawford, S. M., Buckley, D. A. H., Borgnino, J., Blary, F., Nickola, M., and Pickering, T. MNRAS 467, 3699 (2017).

[15] Sarazin, M. and Roddier, F., "The eso differential image motion monitor," Astronomy and Astrophysics, vol. 227, no. 1, Jan. 1990, p. 294-300. (1990).

[16] Avila, R., Ziad, A., Borgnino, J., Martin, F., Agabi, A., and Tokovinin, A., "Theoretical spatiotemporal analysis of angle of arrival induced by atmospheric turbulence as observed with the grating scale monitor experiment," J. Opt. Soc. Am. A 14, 3070-3082 (Nov 1997).

[17] Aristidi, E., Fanteï-Caujolle, Y., Ziad, A., C., D., Chabé, J., and Roland, B., "A new generalized differential image motion monitor," Astronomical Telescopes+ Instrumentation, Proc. SPIE 9145, 91453G (2014).

[18] Aristidi, E., Ziad, A., Chabé, J., Fanteï-Caujolle, Y., Renaud, C., and Giordano, C., "The generalised differential image motion monitor," MNRAS 486, 915 (2019).

[19] Chabé, J., Ziad, A., Aristidi, E., Fanteï-Caujolle, Y., Renaud, C., Blary, F., and Marjani, M., "The calern atmospheric turbulence station," Astronomical Telescopes+ Instrumentation, Proc. SPIE 9906, $99064 Z$ (2016).

[20] Aristidi, E., Fossat, E., Agabi, A., Mékarnia, D., Jeanneaux, F., Bondoux, E., Challita, Z., Ziad, A., Vernin, J., and Trinquet, H., "Dome c site testing: surface layer, free atmosphere seeing, and isoplanatic angle statistics," A \&3 A 499, 955 (2009).

[21] Ziad, A., Aristidi, E., Agabi, A., Borgnino, J., Martin, F., and Fossat, E., "First statistics of the turbulence outer scale at dome c," A\&A 491, 917 (2008).

[22] Racine, R. Publ. Astron. Soc. Pac. 108, 372 (1996).

[23] Martin, F., Tokovinin, A., Ziad, A., Conan, R., Borgnino, J., Avila, R., Agabi, A., and Sarazin, M., "First statistical data on wavefront outer scale at la silla observatory from the gsm instrument," Astronomy and Astrophysics 336, L49-L52 (1998). 